\title{
Projeto e Implementação de Controle em Tempo Real por Alocação de Polos Baseado em Observadores de Estado via Instrumentação Virtual de Alto Desempenho
}

\author{
Lorena Costa de Alencar ${ }^{1}$ \\ Departamento de Eletroeletrônica, IFMA, São Luís, MA, \\ Ginalber Luiz de Oliveira Serra ${ }^{2}$ \\ Departamento de Eletroeletrônica, IFMA, São Luís, MA
}

Resumo. Neste artigo, é proposta uma metodologia de controle por alocação de polos baseado em observadores de estado. Esta metodologia será aplicada a um sistema térmico, projetada e implementada para controle de temperatura, em tempo real, por uma plataforma de aquisição de dados baseada em instrumentação virtual/eletrônica de alto desempenho (LabView).

Palavras-chave. Observadores de Estados, Alocação de Polos, LabView, Identificação no Espaço de Estados

\section{Introdução}

Em vários sistemas de controle, é inviável tanto fisicamente quanto economicamente a instalação dos sensores que serão necessários para medir todas as variáveis de estado. Contudo, com o uso de projeto de sistemas de controle usando observadores de estado é possível reconstruir os estados não medidos ou os valores provenientes de pontos de difícil acesso no sistema, tendo como condição necessária para esta reconstrução apenas que todos os estados sejam observáveis [5].

As técnicas de controle por alocação de polos e de estimação de estados, por meio de observadores, serão aqui combinadas e aplicadas a um sistema térmico, fazendo-se sua identificação a partir dos dados experimentais de entrada e saída, ou seja, a tensão eficaz (Volts RMS) aplicada e a temperatura (graus Celsius). O comportamento dinâmico do sistema térmico será modelado e validado no espaço de estados. O sistema de controle por alocação de polos por meio do observador de estados será projetado e implementado para controle de temperatura do sistema térmico, em tempo real, por meio de uma plataforma de aquisição de dados baseada em instrumentação virtual/eletrônica de alto desempenho (LabView).

\footnotetext{
${ }^{1}$ lorena_alencar2@hotmail.com

22inalber@ifma.edu.br
} 


\section{Formulação do Problema}

\subsection{Identificação no Espaço de Estados}

Para modelos no espaço de estados, é introduzida a seguinte estrutura paramétrica [4]:

$$
A(\theta)=\left[\begin{array}{cc}
0 & 1 \\
X & X
\end{array}\right], B(\theta)=\left[\begin{array}{l}
X \\
X
\end{array}\right], K(\theta)=\left[\begin{array}{l}
X \\
X
\end{array}\right] \quad \text { e } \quad C(\theta)=\left[\begin{array}{ll}
0 & 1
\end{array}\right]
$$

onde:

$\mathrm{X}=$ Parâmetro a ser estimado na identificação.

\subsection{Controle por Alocação de Polos Baseado em Observadores de Esta- dos}

Neste artigo, o problema de controle por alocação de polos baseado em observadores de estados apresenta a seguinte configuração:

$$
\begin{gathered}
\dot{x}=A x+B u \\
y=C x \\
u=-K x+K_{I} \xi \\
\dot{\xi}=r-y=r-C x
\end{gathered}
$$

onde:

$\xi=$ sinal de saída do integrador

$r=$ sinal de entrada de referência

Dada a entrada de referência $\mathrm{r}(\mathrm{t})$ aplicada em $t=0$, para $t>0$, as equações acima podem ser combinadas e descritas sob a forma de uma única equação matricial:

$$
\left[\begin{array}{c}
\dot{x}(t) \\
\dot{\xi}(t)
\end{array}\right]=\left[\begin{array}{cc}
A & 0 \\
-C & 0
\end{array}\right]\left[\begin{array}{c}
x(t) \\
\xi(t)
\end{array}\right]+\left[\begin{array}{c}
B \\
0
\end{array}\right] u(t)+\left[\begin{array}{l}
0 \\
1
\end{array}\right] r(t)
$$

À medida que $t$ tende para infinito pode-se escrever:

$$
\left[\begin{array}{c}
\dot{x}(\infty) \\
\dot{\xi}(\infty)
\end{array}\right]=\left[\begin{array}{cc}
A & 0 \\
-C & 0
\end{array}\right]\left[\begin{array}{l}
x(\infty) \\
\xi(\infty)
\end{array}\right]+\left[\begin{array}{c}
B \\
0
\end{array}\right] u(\infty)+\left[\begin{array}{l}
0 \\
1
\end{array}\right] r(\infty)
$$

Considerando que a entrada $\mathrm{r}(\mathrm{t})$ é suficientemente lenta para $t>0$, subtraindo-se a equação (7) da equação (6), obtém-se:

$$
\left[\begin{array}{c}
\dot{x}(t)-\dot{x}(\infty) \\
\dot{\xi}(t)-\dot{\xi}(\infty)
\end{array}\right]=\left[\begin{array}{cc}
A & 0 \\
-C & 0
\end{array}\right]\left[\begin{array}{c}
x(t)-x(\infty) \\
\xi(t)-\xi(\infty)
\end{array}\right]+\left[\begin{array}{c}
B \\
0
\end{array}\right][u(t)-u(\infty)]
$$

Assim, a equação (8) pode ser escrita da forma:

$$
\left[\begin{array}{c}
\dot{x}_{e}(t) \\
\dot{\xi}_{e}(t)
\end{array}\right]=\left[\begin{array}{cc}
A & 0 \\
-C & 0
\end{array}\right]\left[\begin{array}{c}
x_{e}(t) \\
\xi_{e}(t)
\end{array}\right]+\left[\begin{array}{c}
B \\
0
\end{array}\right] u_{e}(t)
$$


onde

$$
\begin{gathered}
u_{e}(t)=-K x_{e}(t)+K_{I} \xi_{e}(t) \\
e(t)=\left[\begin{array}{l}
x_{e}(t) \\
\xi_{e}(t)
\end{array}\right]
\end{gathered}
$$

As equações (9) e (10) resultam, respectivamente em:

$$
\begin{gathered}
\dot{e}(t)=\hat{A} e+\hat{B} u_{e} \\
u_{e}=-\hat{K} e
\end{gathered}
$$

onde

$$
\hat{A}=\left[\begin{array}{cc}
A & 0 \\
-C & 0
\end{array}\right], \hat{B}=\left[\begin{array}{c}
B \\
0
\end{array}\right] \quad \text { e } \quad \hat{K}=\left[\begin{array}{ll}
K & -K_{I}
\end{array}\right]
$$

A equação de estado de erro substituindo-se a equação (13) na (12):

$$
\dot{e}=(\hat{A}-\hat{B} \hat{K}) e
$$

No sistema de controle por retroação de estado observado, a equação do observador é:

$$
\dot{\widetilde{x}}=\left(A-K_{e} C\right) \widetilde{x}+B u+K_{e} y
$$

E a ação de controle se torna

$$
u=-K \widetilde{x}+K_{I} \xi
$$

\section{Discussões e Resultados}

\subsection{Plataforma de Controle Virtual/Eletrônica}

Neste trabalho, o processo de aquisição de dados para identificação bem como a implementação do método de controle proposto são realizados em uma plataforma de controle virtual/eletrônica desenvolvida com base no software LabVIEW (Laboratory Virtual Instrument Engineering Workbench) e na controladora CompactRIO 9073, ambos da National Instruments. Os principais módulos integrados na controladora e utilizados na plataforma são: NI 9219(entrada analógica) e NI 9263 (saída analógica). Dado que a aplicação será realizada em um processo térmico, a plataforma conta ainda com o sensor de temperatura LM 35. O qual fornece uma tensão proporcional à temperatura a qual está submetido $\left(10 \mathrm{mV} /{ }^{\circ} \mathrm{C}\right)$ e um circuito atuador baseado no circuito integrado TCA 785, capaz de variar o ângulo de disparo de tiristores pela aplicação de uma tensão contínua [6], conforme mostrado na figura 1. 


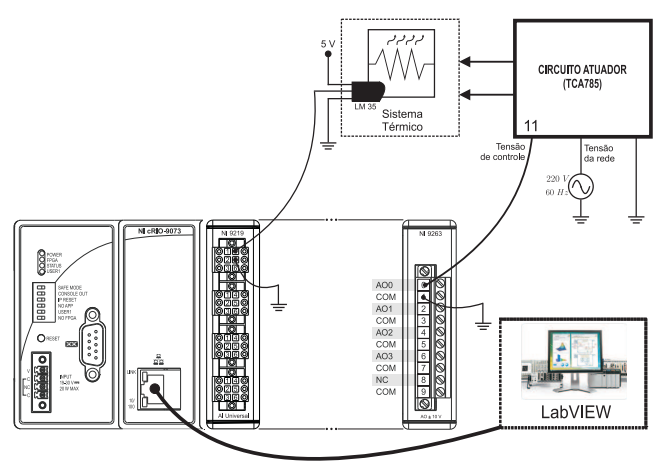

Figura 1: Diagrama esquemático da plataforma de controle virtual/eletrônica de alto desempenho.

\subsection{Identificação do Processo Térmico}

Esta etapa de identificação está baseada em dados experimentais de entrada e saída. Na figura 2 é mostrado o sinal de tensão eficaz (Vrms) aplicado à entrada do sistema e a respectiva saída (Temperatura em ${ }^{\circ} \mathrm{C}$ ) obtida.
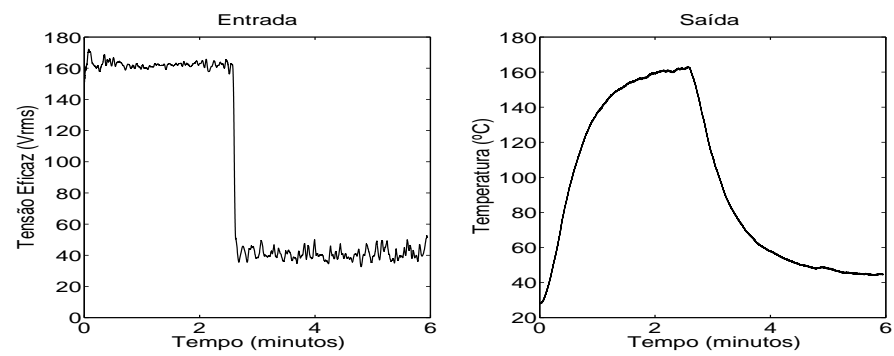

Figura 2: Conjunto de dados de entrada e saída do processo térmico. A tensão alternada elucidada pelo seu valor eficaz, é aplicada ao processo cuja resposta temporal é temperatura, em graus Celsius.

Este conjunto de dados, constituído de 21001 pontos a um tempo de amostragem de 17 milisegundos, foi utilizado para com o auxílio dos métodos de identificação anteriormente comentados obter as seguintes matrizes no espaço de estados:

$$
A=\left[\begin{array}{cc}
0 & 1 \\
-0.0212 & -0.7869
\end{array}\right], B=\left[\begin{array}{c}
0 \\
-0.0244
\end{array}\right], C=\left[\begin{array}{ll}
1 & 0
\end{array}\right] \quad \text { e } \quad D=[0]
$$

Na figura 3 é mostrada a validação do modelo obtido para o processo térmico. O atraso puro de tempo do processo térmico foi obtido aplicando-se correlação cruzada nos dados de entrada e saída, a partir do ponto de máximo do gráfico (valor de 1949), com o tempo de amostragem de 17 milissegundos, fornecendo $\tau_{d}=3.3133 \mathrm{~s}$. 


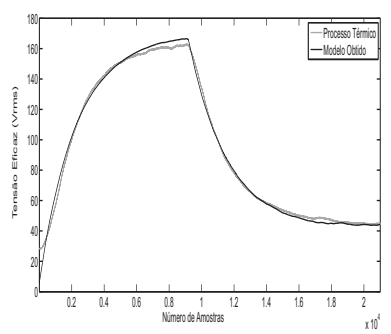

(a) Validação do modelo obtido por identificação no espaço de estados.

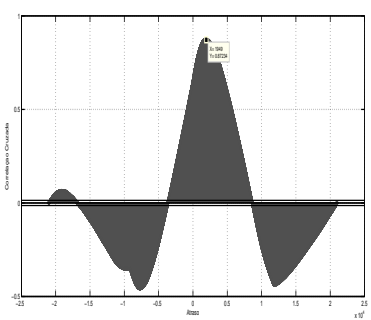

(b) Função de correlação cruzada para estimação do atraso puro de tempo.

Figura 3: Dados da validação do modelo e estimação do atraso puro de tempo.

\subsection{Implementação da Metodologia de Controle e Estimação de Estados}

A metodologia de controle por alocação de polos aliada ao conceito de observadores de estados foi computacionalmente desenvolvida na plataforma MATLAB/SIMULINK e aplicada ao processo térmico com o auxílio da plataforma de controle virtual/eletrônica Labview.

Foram escolhidos os polos $-17.6248 \mathrm{e}-0.3752$ por meio da determinação de parâmetros de resposta temporal como o tempo de acomodação e coeficiente de amortecimento. Em seguida, foram determinadas a matriz de ganho de retroação de estados, o ganho do integrador e a matriz do ganho do observador, expressas na equação (19).

$$
K=\left[\begin{array}{ll}
6.46 & 8.22
\end{array}\right], K_{I}=37.6547 \text { e } \quad K_{e}=\left[\begin{array}{c}
-284.53 \\
705.48
\end{array}\right]
$$

Os principais resultados obtidos são comparados na figura 4 a fim de demonstrar a eficiência da metodologia proposta.
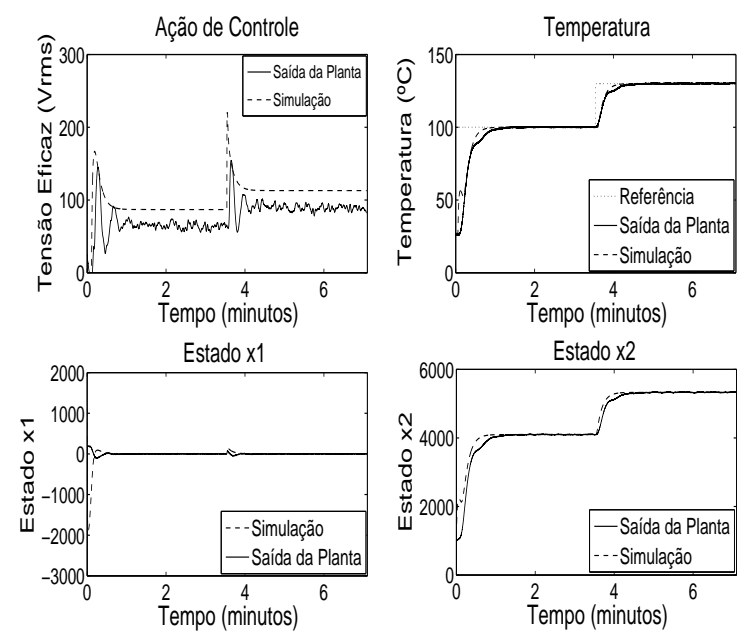

Figura 4: Comparação entre respostas da Planta e da simulação 
Os resultados obtidos levam a uma resposta com tempo de acomodação de 1.41 minutos sem ocorrência de sobressinal e ilustram que o controle ocorreu de forma eficiente, baseado em polos bem posicionados. Pode ser observado que os estados foram estimados de maneira coerente. Portanto, trata-se de uma metodologia estável e com boas respostas tanto em simulação analógica quanto em aplicações de controle em tempo real.

\section{Conclusões}

Conforme proposto, foi realizada a implementação de uma estratégia de controle por alocação de polos baseada em observadores de estados em tempo real utilizando instrumentação virtual/eletrônica de alto desempenho. Com os resultados experimentais procura-se evidenciar a flexibilidade e eficiência da metodologia proposta para a estimação dos estados e controle do sistema de acordo com o desempenho desejado pelo projetista.

\section{Agradecimentos}

Os autores agradecem ao Conselho Nacional de Desenvolvimento Científico e Tecnológico $(\mathrm{CNPq})$ e à Fundação de Amparo à Pesquisa e Desenvolvimento Científico do Maranhão (FAPEMA) pelo fomento à pesquisa.

\section{Referências}

[1] L. Bin, A new optimized pole placement strategy of grid-connected inverter with LCL-filter based on state variable feedback and state observer, Applied Power Electronics Conference and Exposition (APEC), Twenty-Eighth Annual IEEE , vol., no., pp.2900,2906, (2013).

[2] C.H. Choi, Step Response Improvement by Pole Placement with Observer, System Theory, 40th Southeastern Symposium on , vol., no., pp.7,12, (2008).

[3] W. Lei, Design of autopilot with Pole placement and State-Observer Feedback, Computer Science and Automation Engineering (CSAE), IEEE International Conference on , vol.4, no., pp.53,56, (2011).

[4] L. Ljung, System Identification - Theory For the User, 2nd ed, PTR Prentice Hall, Upper Saddle River, N.J., (1999).

[5] D. G. Luenberger, Observing the state of a linear system. IEEE Military Eletronics, New York, (1964).

[6] R. J. M. Santos, Controle Fuzzy Multiobjetivo de Sistemas Dinâmicos Não-Lineares com Atraso Puro de Tempo, Proceeding Series of the Brazilian Society of Computational and Applied Mathematics, vol. 1, (2013), DOI: 10.5540/03.2013.001.01.0178. 
[7] R. Schmid, Robust Pole Placement With Moore's Algorithm, Automatic Control, IEEE Transactions on , vol.59, no.2, pp.500,505, (2014) 